\title{
NIVELES DE DESARROLLO DEL LENGUAJE COMPRENSIVO EN NIÑOS DE SEGUNDO A SEXTO GRADO DE PRIMARIA
}

\author{
Violeta Tapia Mendieta*
}

\section{RESUMEN}

Se analizan y describen las características de los niveles de desempeño del lenguaje de una muestra de 541 escolares de segundo a sexto de primaria procedentes de centros educativos estatales y particulares de Lima Metropolitana y se presentan las normas percentilares de las áreas y subtests de la Batería Woodcock de Proficiencia en el idioma en función a la edad y grado escolar.

Palabras clave:

Batería Woodcock de Proficiencia en el idioma, normas percentilares, lenguaje.

\begin{abstract}
The article analizes the quantitative characteristics of the language proficiency on a sample of 541 elementary students from second to sixth degree of Lima Metropolitana. Percentile ranks related to the total score, areas and subtests of the Woodcock Language Proficiency Battery are presented.
\end{abstract}

Key words:

Woodcock Proficiency Battery, percentile ranks, language.

Se agradece sinceramente a la Ps. Norma Quispe por su colaboración en la administración de las pruebas y al Ps. Luis Miguel Escurra por el procesamiento computarizado de los datos 
El presente informe representa la ampliación de un estudio que diéramos a conocer en una publicación anterior sobre la estandarización de la Batería Woodcock de Proficiencia en el Idioma (Tapia, 1998), el que incluyó la adaptación cultural de algunos ítemes, análisis psicométrico del instrumento y la elaboración de normas percentilares globales por edad y grado en estudiantes de 2do. a 6to. grado de educación primaria.

Con el propósito de describir las características del desarrollo del lenguaje que presentan los niños y, contar con baremos parara elaboración de perfiles diferenciales de proficiencia lingüística de las áreas del lenguaje y de las habilidades que mide cada una de las subpruebas de la Batería, juzgamos necesario e importante ampliar la muestra así como las normas de interpretación.

Nuestro interés en el estudio se justifica por la importancia del desarrollo del lenguaje como requisito esencial para el éxito académico y por ser además, un medio de comunicación, expresión de desarrollo cognitivo y afectivo, de control y regularización del comportamiento humano. Se parte del presupuesto que la actividad o comportamiento lingüístico humano está apoyado en el conocimiento del lenguaje que como tal se encuentra representado en la mente de todo hablante.

Pese a la indudable polisemia del término "lenguaje", es posible entrever ciertas regularidades: a) que el lenguaje puede interpretarse como un sistema compuesto por unidades lingüísticas cuya organización interna puede ser objeto de una descripción estructural formal; b) el hecho que la adquisición y uso del lenguaje por parte de los organismos posibilita en éstos, formas peculiares y específicas de relación y acción sobre el medio (especialmente sobre el medio social); y e) el hecho que el lenguaje se materializa y da lugar a formas concretas de conducta, lo que permite interpretarlo como modalidad de comportamiento.

Hablar de lenguaje, sea cual sea la naturaleza o modalidad perceptiva de sus unidades constituyentes, implica siempre hablar de la existencia de un conjunto de signos (dimensión estructural). Tal y como tradicionalmente se ha destacado desde una perspectiva semiótica, el lenguaje puede ser interpretado, en primer lugar como un sistema de elementos (señales, signo, símbolos) que resulta relevantes para un organismo porque remite a objetos o aspectos de la realidad distintos a ellos mismos.

En tanto que sistema formado por signos, el lenguaje humano puede ser objeto de descripciones semánticas sintácticas y pragmáticas. Para casi todos los investigadores, el lenguaje es el sistema prototipo de simbolización, cuyo desarrollo se realiza en base a destrezas léxicas, sintácticas y semánticas. En efecto, el lenguaje se constituye con tres aspectos centrales: 1) las reglas que rigen la ordenación y la organización del sistema simbólico (gramática o sintaxis del sistema) 2) los significantes explícitos o las denotaciones de los símbolos y los objetos, las ideas o los "referentes" a los que se refieren los símbolos (del sistema simbólico), 3) los usos y las funciones de los símbolos (la pragmática del sistema).

La adquisición y desarrollo de signos van ligados a la realización de actividades tales como la comunicación y la interacción social, la expresión emocional y también, en la especie humana, a la conducta voluntaria y el pensamiento racional (dimensión funcional).

Hablar de lenguaje implica también considerado como un tipo de comportamiento, 
actividad (dimensión conductual). Dicho comportamiento es aquel que realizan el emisor y el receptor, cuando respectivamente, codifican y producen o reciben, decodifican $e$ interpretan mensajes lingüísticos mediante la utilización de un código de signos o símbolos común y compartido por ambos. Como conducta o actividad que es, el lenguaje adopta dos modalidades primarias o básicas, la producción y la comprensión. Estas dos modalidades en última instancia, se realizan en forma simultánea y combinadas durante la conversación.

Tomadas en su conjunto, las tres dimensiones, formal, funcional y comportamental permiten precisar de forma sustancial el concepto de lenguaje, restringiendo el uso de este término a "los sistemas de expresión, representación y comunicación que se basa en sistema de signos formalmente bien definidos y cuya utilización implica una modalidad de comportamiento". (Belinchon, Riviere e Igoa (1992).

Desde una perspectiva funcional, la actividad lingüística es en realidad, una actividad cooperativa, que implica intercambios de objetos, intenciones (tales como creencias, conocimientos y deseos). El lenguaje presupone y requiere el empleo de procesos mentales que no sólo norman sobre el lenguaje mismo sino también los objetos a los que refiere y las personas a las que se dirige. En esta dimensión es importante destacar la función representacional unida a la simbólica, lo cual otorga al lenguaje una función cognitiva básica que estimula los procesos de abstracción y categorización.

En términos de Vygotsky (1988), "el lenguaje combina la función comunicativa con la de pensar. Su función comunicativa es el sustrato que hace posible inicialmente, una actividad intelectual inter-psicológica, proceso que culminará por el dominio completamente interiorizado de la nueva función psicológica, mediada por signos. La unión entre pensamiento y lenguaje, en forma más simple es el significado de la palabra".

En el contexto de la Batería Woodcock de Proficiencia en el Idioma, (BWPI) el dominio del lenguaje está referido a las habilidades del lenguaje oral, comprensión lectora y el lenguaje escrito que en su conjunto conformaran el lenguaje comprensivo.

La BWPI en su versión es español fue estandarizada bajo la dirección de su autor, Richard Woodcock en una muestra de 800 sujetos provenientes de países de habla hispana: Costa Rica, Puerto Rico, Perú, México y España.

El uso de la batería ha demostrado su utilidad para fines de evaluación di agnóstica. Tapia (1995), aplicó el instrumento en un grupo de niños deficientes y buenos lectores contrastándolos con una prueba de comprensión lectora. Se comprobó su validez concurrente, sumándose a la validez de constructo a través del análisis factorial.

Asimismo, se determinó su confiabilidad por el método de di visión por mitades. Aún cuando la estandarización original incluyó una muestra del Perú, para validación, se han realizado adaptaciones socioculturales en relación a informaciones, objetos y expresiones lingüísticas, así como corrección del significado de algunos términos. 
Los objetivos principales del presente estudio son:

- Describir las características del desarrollo del lenguaje comprensivo de los alumnos de segundo a sexto grado de Primaria, considerando el grado escolar y la edad cronológica.

- Desarrollar las normas de interpretación de la Batería Woodcock de Proficiencia en el Idioma de las áreas y de los subtests que la conforman por grado escolar y edad.

\section{METODO}

\section{Muestra Normativa}

Estuvo integrada por 541 escolares de segundo a sexto grados de Primaria, representativa de la población de centros educativos estatales y particulares de Lima Metropolitana. Las variables consideradas fueron:

Edad: Se tomó en cuenta el año completo de cada edad a partir de la fecha de nacimiento hasta última fecha de cumpleaños, lo cual significa que no se consideraron los meses adicionales ( 1 a 11 meses) comprendidos entre el último cumpleaños y la fecha de aplicación de la prueba.

El rango de edad de la muestra osciló entre 6 a 13 años. Por existir pocos casos de edades extremas, los alumnos de 6 años integraron la muestra de 7 y, los de 12 y 13, la muestra de 11 años.

Sexo: Cada grado estuvo representado por escolares de ambos sexos. La muestra total incluye 360 varones y 181 mujeres.

Grado Escolar: Se consideró el grado escolar que cursaban los alumnos a partir del mes de Junio, debido que los meses de Abril y Mayo son períodos de adaptación.

Los alumnos evaluados en los dos primeros meses escolares fueron incluidos en el grado inmediato inferior.

Nivel Socioeconómico: De acuerdo al tipo de plantel de procedencia, la muestra comprendió 410 alumnos de centros educativos estatales y 131 particulares. $N^{\circ} 1$

La distribución de la muestra según la edad y el grado escolar aparecen en la Tabla

Tabla $\mathbf{N}^{\circ} \mathbf{1}$

Distribución de la muestra por edad y grado

\begin{tabular}{|c|ccccc|c|}
\hline G. Esc. & \multicolumn{5}{|c|}{ Grado escolar } & \multirow{2}{*}{ Total } \\
\hline & $\mathbf{2}$ & $\mathbf{3}$ & $\mathbf{4}$ & $\mathbf{5}$ & $\mathbf{6}$ & \\
\hline $\mathbf{7}$ & 55 & 3 & & & & 10.7 \\
$\mathbf{8}$ & 49 & 38 & 8 & & & 17.6 \\
$\mathbf{9}$ & 8 & 44 & 55 & 11 & & 21.8 \\
$\mathbf{1 0}$ & 2 & 12 & 41 & 68 & 5 & 26.2 \\
$\mathbf{1 1}$ & & 2 & 10 & 31 & 99 & \\
\hline \multirow{2}{*}{ Total } & 114 & 99 & 114 & 110 & 104 & 541 \\
& 21.4 & 18.3 & 21.1 & 20.3 & 19.2 & 100 \\
\hline
\end{tabular}




\section{Descripción de la Batería Woodcock de Proficiencia en el Idioma (BWPI)}

La BWPI comprende ocho subtests agrupados en tres áreas o dimensiones del dominio del lenguaje: Lenguaje Oral, Lectura y Escritura. En la tabla $\mathrm{N}^{\circ} 2$ se describe la Batería: el código y nombre de cada subtest, el número de ítems que contiene y lo que mide.

Tabla $\mathbf{N}^{\circ} 2$

Subtests de la BWPI

\begin{tabular}{|c|c|c|}
\hline Subtests & $\begin{array}{l}\text { Número } \\
\text { de Items }\end{array}$ & Mide \\
\hline $\begin{array}{l}\text { Lenguaje oral } \\
\text { A. Vocabulario de dibujos }\end{array}$ & 33 & $\begin{array}{l}\text { Identificar por su nombre dibujos de objetos } \\
\text { y acciones }\end{array}$ \\
\hline B. Antónimos - Sinónimos & 57 & $\begin{array}{l}\text { Conocimiento del significado de palabras } \\
\text { vinculado a la conceptualización verbal }\end{array}$ \\
\hline C. Analogías & 37 & $\begin{array}{l}\text { Razonamiento analógico mediante } \\
\text { razonamiento inductivo }\end{array}$ \\
\hline $\begin{array}{l}\text { Lectura } \\
\text { D. Identificación de palabras }\end{array}$ & 50 & Lectura correcta de letras y palabras \\
\hline E. Análisis de palabras & 28 & $\begin{array}{l}\text { Aplicación de destrezas de análisis fonético } \\
\text { y estructural al pronunciar palabras no } \\
\text { familiares }\end{array}$ \\
\hline F. Comprensión de textos & 28 & $\begin{array}{l}\text { Habilidad para analizar un texto corto e } \\
\text { identificar una palabra omitida }\end{array}$ \\
\hline $\begin{array}{l}\text { Lenguaje escrito } \\
\text { G. Dictado }\end{array}$ & 42 & $\begin{array}{l}\text { Habilidades ortográficas, puntación y } \\
\text { empleo de mayúsculas, uso del tiempo de los } \\
\text { verbos y plural de las palabras }\end{array}$ \\
\hline H. Comprobación & 40 & $\begin{array}{l}\text { Estrategias metacomprensivas a través de la } \\
\text { identificación de errores en textos y } \\
\text { conocimientos sobre como corregirlos }\end{array}$ \\
\hline
\end{tabular}

\section{Validez y Confiabilidad}

En el informe anterior (Tapia, 1998) se dio a conocer los resultados de los estudios realizados sobre la validez y confiabilidad que le otorgan la justificación estadística a la BWPI para su utilización en nuestro medio.

Siguiendo las recomendaciones de APA, con la muestra ampliada se ha efectuado un estudio de la validez de constructo a través de un análisis factorial comprensivo exploratorio (Browne, Cudeck,Tateneni y Mels,1998). 
Tabla $\mathbf{N}^{0} 3$

Matriz de Correlación entre los Subtests

\begin{tabular}{|c|c|c|c|c|c|c|c|c|}
\hline & A & B & C & D & E & F & G & H \\
\hline B & 0.71 & & & & & & & \\
\hline C & 0.60 & 0.72 & & & & & & \\
\hline D & 0.29 & 0.41 & 0.46 & & & & & \\
\hline E & 0.19 & 0.26 & 0.34 & 0.64 & & & & \\
\hline F & 0.67 & 0.69 & 0.65 & 0.43 & 0.31 & & & \\
\hline G & 0.49 & 0.59 & 0.62 & 0.54 & 0.42 & 0.60 & & \\
\hline H & 0.58 & 0.67 & 0.70 & 0.53 & 0.10 & 0.69 & 0.71 & \\
\hline
\end{tabular}

Se tomó como fuente de datos la matriz de correlaciones entre los diferentes subtests como se muestra en la siguiente tabla:

El análisis factorial solución oblicua OBLIMIN identificó claramente dos factores en los subtests componentes de la Batería. Dichos factores no son independientes. Las tablas 4 y 5 muestran

Tabla $\mathbf{N}^{\circ} 4$

Análisis Factorial. Solución OBLIMIN de los Subtests

\begin{tabular}{|c|c|c|}
\cline { 2 - 3 } \multicolumn{1}{c|}{} & Factor 1 & Factor 2 \\
\hline $\mathbf{A}$ & .83 & .17 \\
\hline $\mathbf{B}$ & .89 & .32 \\
\hline $\mathbf{C}$ & .85 & .43 \\
\hline $\mathbf{D}$ & .48 & .88 \\
\hline $\mathbf{E}$ & .31 & .90 \\
\hline $\mathbf{F}$ & .86 & .38 \\
\hline $\mathbf{G}$ & .75 & .60 \\
\hline $\mathbf{H}$ & .84 & .55 \\
\hline \% de Varianza & 60.5 & 15 \\
\hline
\end{tabular}

Tabla $\mathbf{N}^{\circ} 5$

Matriz de Correlación entre factores

\begin{tabular}{|l|c|c|}
\cline { 2 - 3 } \multicolumn{1}{c|}{} & Factor $\mathbf{1}$ & Factor $\mathbf{2}$ \\
\hline Factor 1 & 1.000 & \\
\hline Factor 2 & .41 & 1.000 \\
\hline
\end{tabular}

la estructura de la matriz factorial y la matriz de la correlación entre los dos factores, respectivamente:

El primer factor explica el $60.5 \%$ de la varianza total y tiene mayor peso en los subtests Vocabulario de Dibujos, Antónimos-Sinónimos, Comprensión de textos, Dictado y 
Comprobación vinculados al procesamiento lingüístico mas complejo que incluye procesos de categorización, interpretación de significados, relaciones analógicas y meta-comprensión. El segundo factor explica el $15 \%$ de la varianza y vincula los procesos que subyacen a los subtests Identificación de palabras y Análisis de palabras referidos fundamentalmente, a los de decodificación y codificación.

Los dos factores identificados no se excluyen entre sí. Siendo el lenguaje un sistema complejo de comunicación, dichos factores están interrelacionados, con diferentes cargas factoriales en los diferentes subtests.

\section{PRESENTACION Y DISCUSION DE LOS RESULTADOS}

Las tablas 6, 7, 8 y 9 presentan los promedios y las desviaciones estándar de los puntajes globales, los de las áreas y los obtenidos en los diferentes subtests por grado y edad lo que nos permiten realizar dos tipos análisis:

a) Comparaciones de las diferencias entre las medias, para verificar la conformación de los grupos normativos $\mathrm{y}$,

b) Descripción de las características del desarrollo del lenguaje en relación a las variables del estudio.

\section{Significatividad de las diferencias entre las medias}

Las comparaciones se han realizado mediante el análisis de varianza de un factor y de las prueba post hoc Scheffe, habiéndose comprobado la significatividad de las diferencias entre las medias a un nivel de confianza al .001.

Las letras que aparecen junto a los promedios son los códigos de los pares de comparaciones e indican entre que par de valores de cada variable existen diferencias estadísticas significativas; por ejemplo, en la Tabla 6 a indica que existen diferencias en el total general entre 2 do $(M=115.22)$ y 6to grado $(M=171.99)$ y así sucesivamente.

\section{Características de los niveles de desarrollo del lenguaje}

Para hacer los grupos comparables entre sí, los promedios se analizan en términos de porcentajes.

Al considerar la variable grado escolar, se observa en general, un incremento progresivo de los puntajes totales, de las áreas y de los subtests. Los valores promedios globales representan porcentajes que oscilan entre 36\% (segundo grado) y 59\% (sexto) en relación al puntaje total esperado, representado la prueba en su conjunto, un nivel medio de dificultad.

El perfil de rendimiento por áreas, revela un mejor desempeño en Lectura, con niveles que fluctúan entre $61 \%$ a $75 \%$. Lenguaje Oral y Lenguaje Escrito presentan un rango entre $23 \%$ a $38 \%$ y $25 \%$ a $52 \%$, respectivamente. Estas dos áreas resultaron difíciles en segundo grado y de mediana dificultad en los grados superiores.

$\mathrm{Al}$ analizar los promedios de los subtest, se observa que el Lenguaje Oral, los valores son consistente mente bajos en Vocabulario de Dibujos, Antónimos, Sinónimos y Analogías, especialmente en los grados 2do y 3ro -El desempeño en los grados superiores revela que los subtests son de mediana dificultad - Los alumnos en general, presentan deficiencias en el uso de código verbales para identificar dibujos de objetos y acciones, en identificar sinónimos más que antónimos y en razonamiento analógico.

En Lectura, los substest que explican mejor el desempeño del área son los de Lectura 
Oral (Identificación de palabras y Análisis de palabras), que resultaron ser fáciles en todos los grados. El subtests Comprensión de textos muestra un desempeño más bien bajo cuyos promedios representan porcentajes que fluctúan entre $23 \%$ a $41 \%$; difícil para segundo grado y de mediana dificultad en los demás grados. Predomina la comprensión literal sobre la inferencial.

El lenguaje Escrito, el subtests Dictado presenta un mejor nivel de desempeño, representando los promedios, un rango porcentual entre 37\% y 63\% (segundo y sexto grados, respectivamente), representando un nivel de dificultad media. La mayores deficiencias se advierten en ortografía, uso del tiempo de los verbos y signos de puntuación. El subtests Comprobación presenta valores que representan porcentajes mas bien bajos con un rango que varia entre $13 \%$ a $38 \%$. Los promedios no solo los más bajos del área sino de todos los subtests de la Batería. Los alumnos, especialmente los de segundo y tercer grados muestran serias deficiencias en identificar de puntuación, sintaxis, y ortografía.

En relación a la variable edad, los valores promedios son semejantes aunque un poco inferiores a los de grado, a excepción de la edad de 7 años.

El que en cada grado escolar existan alumnos de diferentes edades hace que las dos variables, edad y grado, conformen grupos diferenciados, no siendo posible establecer una correspondencia directa entre ellas. Este hecho tiene implicancias para el diagnóstico psicopedagógico: considerar la experiencia educativa (grado escolar), como base para el diagnóstico no sólo porque la Batería se ha desarrollado por grados, sino porque esta variable influyen más en el rendimiento, como ha sido demostrado en otras investigaciones. Sin embargo, al tomar en cuenta también la edad, se complementa la información pues permite determinar en qué medida el puntaje de un alumno de determinada edad es igualo se desvía de sus coetáneos. Es con esta finalidad, que hemos desarrollado las normas de interpretación en función al grado escolar y edad.

\section{Normas de interpretación}

Se han desarrollado normas percentilares por grado y edad para el puntaje total, los puntajes de las áreas y de los subtests, de modo que permitan la elaboración de perfiles individuales de desempeño.

Las puntuaciones directas se convierten a los respectivos rangos percentilares para obtener información sobre cómo un alumno se compara con otros de su mismo grado o edad. Los puntajes que se ubican en el percentil 50 corresponden al rango normal; los puntajes inferiores al percentil 50 corresponden al rango normal; los puntajes inferiores al percentil 25 corresponden al rango inferior al promedio (bajo) y aquellos alumnos que obtienen puntajes por encima del percentil 75 , demuestran un nivel de desarrollo superior. En una próxima publicación se dará a conocer los baremos respectivos 


\section{BIBLIOGRAFIA}

Anastasi A- y Urbina (1998) Tests Psicológicos. Prentice may, México

Belinchon, M.; Riviere, A.; e Igoa, J. (1992) Psicología del Lenguaje. Investigación y teoría. Edit. Trolta

De Vega M. (1996) Introducción a la Psicología Cognitiva. Edit. Alianza Madrid

Gonzáles, R. (1993) Lectoescritura. Un enfoque cognitivo. Lima CEDHUM

Limusa, 1. (1987) Estructura Lingüísticas Básicas del Español. Edit. Limusa. México

Nunnally, J. Y Bernstein, J.(1995) Teoría Psicométrica. México

Pinzas, J. (1995) Leer Pensando. Una Introducción a la Visión Contemporánea de

Lectura. Serie fundamentos de la lectura. AIAP

Pinzas, J. (1997) Metacognición y Lectura. PUCP

Tapia, V. (1995) Desarrollo de un programa de Lectura en Niños Deficientes Lectores. Informe Final. USMMSM

Tapia, V. (1998) Estandarización de la Batería Woodcock de Proficiencia en el Idioma. Revista de Investigación en Psicología UPSI UNMSM

Tapia, V. (1999) Efectos de un Programa de Estrategias Cognitivas y Metacognitivas para mejorar la Comprensión Lectora. Revista de Psicología. Facultad de Psicología UNMSM

Titone, R. (1985) Psicolinguística aplicada. Introducción a la didáctica del lenguaje. Madrid. Ed. Kapeluz

Vygotsky, L.S. (1997) Pensamiento y Lenguaje. Ed. La Pleyade. BS Aires

Vygotsky, L.S (1998) El desarrollo de las funciones Psicológicas Superiores. Edición a cargo de CoJe, M.; John Steiner., Scribner, S. y Ouberman E. Edil. Grijalbo Barcelona Woodcock, R- (1981) Woodcok Language Proficiency Battery. Spanish Form. USA. 
Tabla $\mathbf{N}^{\circ} 6$

Análisis de Varianza de un factor del Puntaje y por áreas de la Batería de Woodcock según grado de estudios

\begin{tabular}{|c|c|c|c|c|c|c|c|c|c|c|c|}
\hline \multirow{2}{*}{$\begin{array}{r}\text { Grado de } \\
\text { Estudios }\end{array}$} & \multicolumn{2}{|c|}{$\begin{array}{c}2^{\circ} \\
N=114\end{array}$} & \multicolumn{2}{|c|}{$\begin{array}{c}3^{\circ} \\
N=99\end{array}$} & \multicolumn{2}{|c|}{$\begin{array}{c}4^{\circ} \\
N=114\end{array}$} & \multicolumn{2}{|c|}{$\begin{array}{c}5^{\circ} \\
N=110\end{array}$} & \multicolumn{2}{|c|}{$\begin{array}{c}6^{\mathbf{o}} \\
\mathrm{N}=104\end{array}$} & \multirow{2}{*}{$\mathbf{F}$} \\
\hline & $\mathbf{M}$ & DS & $\mathbf{M}$ & DS & $\mathbf{M}$ & DS & $\mathbf{M}$ & DS & $\mathbf{M}$ & DS & \\
\hline Total General & $1115.22_{\text {adgi }}$ & 24.28 & $138.89_{\text {behi }}$ & 26.39 & $151.53_{\text {cfgh }}$ & 26.95 & $171.41_{\text {def }}$ & 25.83 & $171.99_{\mathrm{abc}}$ & 23.91 & $96.47^{* * *}$ \\
\hline Lenguaje Oral & $29.98_{\text {adfg }}$ & 9.50 & $39.54_{\text {beg }}$ & 9.90 & $44.30_{\mathrm{cf}}$ & 12.88 & $50.02_{\mathrm{abc}}$ & 12.27 & $48.84_{\mathrm{de}}$ & 12.55 & $5.52 * * *$ \\
\hline Lectura & $64.67_{\text {adgh }}$ & 11.19 & $70.57_{\text {beh }}$ & 11.30 & $72.61_{\mathrm{cfg}}$ & 10.01 & $79.88_{\text {def }}$ & 7.90 & $80.41_{\mathrm{abc}}$ & 7.28 & $51.43^{* * * *}$ \\
\hline $\begin{array}{l}\text { Lenguaje } \\
\text { Escrito }\end{array}$ & $20.58_{\text {adgi }}$ & 8.03 & $28.79_{\text {behi }}$ & 9.86 & $34.61_{\text {cfgh }}$ & 9.27 & $41.51_{\text {def }}$ & 9.80 & $42.64_{\mathrm{abc}}$ & 8.67 & $111.25^{* * *}$ \\
\hline
\end{tabular}

$* p<.05, p<.01 m * * * p<.001$

Gl $1=4$

Gl $2=536$

Comparaciones significativas con el test de Scheffé: $a, b, c, d, e, f, g, h, i$ 
Tabla $\mathbf{N}^{\circ} 7$

Análisis de Varianza de un factor de los Subtests de la Batería de Woodcock según grado de estudios

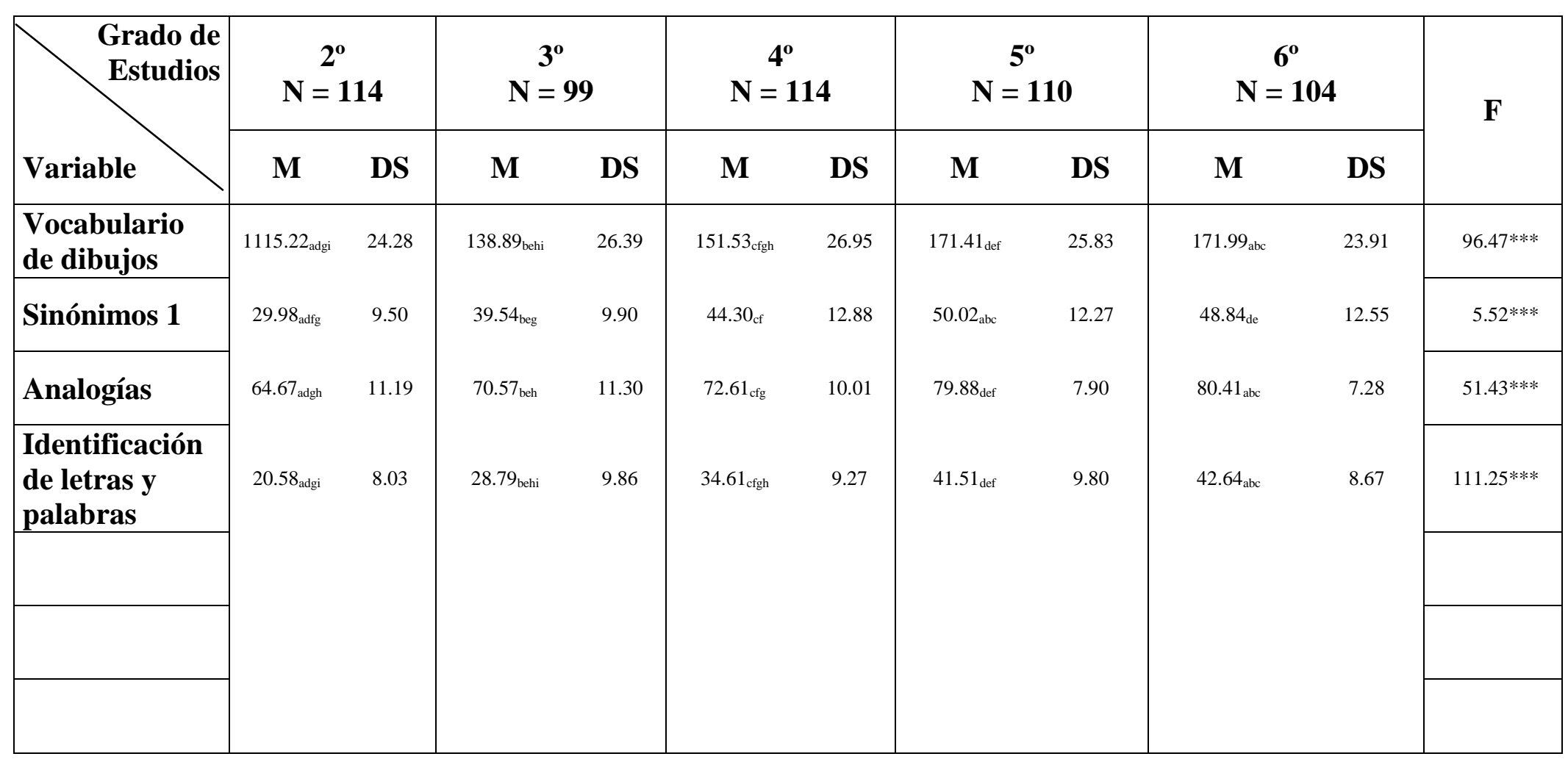

$* p<.05, p<.01 m * * * p<.001$

Gl $1=4$

Gl $2=536$

Comparaciones significativas con el test de Scheffé: $a, b, c, d, e, f, g, h, i$ 